\title{
Ductal carcinoma in situ (DCIS): pathological features, differential diagnosis, prognostic factors and specimen evaluation
}

\author{
Sarah E Pinder \\ Breast Research Pathology, Research Oncology, Division of Cancer Studies, King's College London, \\ Guy's Hospital, London, UK
}

\begin{abstract}
Ductal carcinoma in situ (DCIS) is a heterogeneous, unicentric precursor of invasive breast cancer, which is frequently identified through mammographic breast screening programs. The lesion can cause particular difficulties for specimen handling in the laboratory and typically requires even more diligent macroscopic assessment and sampling than invasive disease. Pitfalls and tips for macroscopic handling, microscopic diagnosis and assessment, including determination of prognostic factors, such as cytonuclear grade, presence or absence of necrosis, size of the lesion and distance to margins are described. All should be routinely included in histopathology reports of this disease; in order not to omit these clinically relevant details, synoptic reports, such as that produced by the College of American Pathologists are recommended. No biomarkers have been convincingly shown, and validated, to predict the behavior of DCIS till date.

Modern Pathology (2010) 23, S8-S13; doi:10.1038/modpathol.2010.40
\end{abstract}

Keywords: ductal carcinoma in situ (DCIS); breast cancer; histopathology; prognostic factors

Ductal carcinoma in situ (DCIS) is a malignant, clonal proliferation of cells growing within the basement membrane-bound structures of the breast and with no evidence of invasion into surrounding stroma. Where mammographic breast screening is not performed, DCIS constitutes approximately $5 \%$ of breast cancers but within screening programs it comprises approximately $20-25 \%$ of these tumors. However, it is now generally accepted that DCIS is not one entity but rather a heterogeneous group of lesions clinically, radiologically, morphologically and genetically. This heterogeneity adds complexity to the pathologist's role, in macroscopic specimen handling, and microscopic diagnosis and prognostic feature assessment.

\section{Specimen handling}

This clinical treatment of DCIS is surgical excision with clear margins. ${ }^{1}$ For large lesions this may require mastectomy but for smaller, screen-detected

Correspondence: Dr SE Pinder, Breast Research Pathology, Division of Cancer Studies, King's College London, 3rd Floor, Bermondsey Wing, Guy's Hospital, Great Maze Pond, London SE1 9RT, UK.

E-mail: Sarah.pinder@kcl.ac.uk

Received 19 January 2010; accepted 19 January 2010 lesions, a good cosmetic result can be obtained by wide local excision. Recurrence of DCIS generally occurs at the site of previous excision and it is therefore better regarded as residual disease, as demonstrated by studies showing concordance of genetic pattern with comparative genomic hybridization (CGH) in primary and recurrent lesions. ${ }^{2}$ In addition, approximately half of the recurrences after breast-conserving surgical excision for DCIS are as invasive disease, ${ }^{3}$ with potential to spread outside of the breast. Thus, complete surgical excision with accurate pathological assessment and reporting is essential to optimize treatment of a curable in situ process that may otherwise progress into, or recur as, invasive carcinoma.

Although clear margins of excision of DCIS are clearly important prognostically, with regard to local recurrence of disease, ${ }^{4}$ there is, no widely agreed margin width of clearance at which the DCIS is deemed 'completely excised'. If DCIS is excised by $1 \mathrm{~mm}$ or less, local recurrence is more common, as is residual disease in a subsequent re-excision specimen or completion mastectomy. ${ }^{5-8}$ However, a range of minimum width of surrounding uninvolved tissue is recommended in different centers, from 2 (with radiotherapy) to $10 \mathrm{~mm}$ or more being regarded as adequate clearance. This lack of a uniform definition for 'complete' excision has implications 


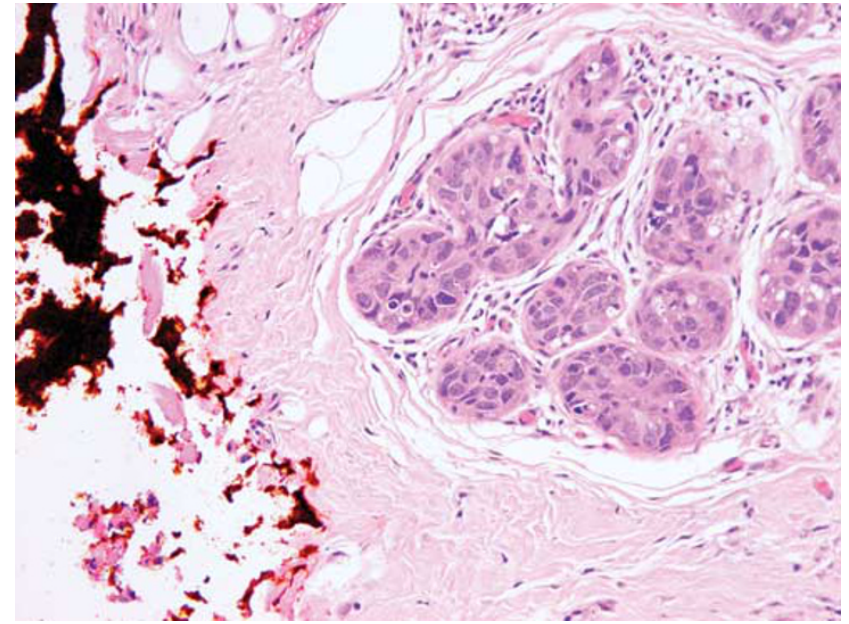

Figure 1 High-grade ductal carcinoma in situ (DCIS) seen as cancerization of lobules present less than $1 \mathrm{~mm}$ from the lateral margin of this wide local excision specimen (seen as orange ink).

with regard to handling of the surgical excision specimen within the laboratory.

Ductal carcinoma in situ is most commonly surgically excised on the basis of the placement of needle localization wires by the radiologist, who may bracket the lesion with more than one wire. Thus, the specimen is received bearing one or more wires in the histology laboratory. It should be weighed and measured. The surgeon should orientate the specimen with clips or sutures according to local protocol and different margins can then be painted with marker inks. These allow identification of the different margins histologically (Figure 1), such that if disease does extend to one margin, this aspect alone can be re-excised, without resorting to excision of the entire cavity. On subsequent slicing, either in the fresh state or after fixation, there may be prominent fibrous tissue around the ducts or, small specks of necrotic material may extrude from the cut ends of the ducts, although commonly no macroscopic abnormality is seen. This absence of gross features makes selection of blocks challenging. Slicing and X-ray of each specimen slice is generally required to find and target the microcalcification with which the disease has presented.

Of particular relevance, therefore, is the finding that not all of the DCIS may be visible on the X-ray; $85 \%$ of the area may be seen mammographically for comedo/solid DCIS but only $50 \%$ of the area of micropapillary/cribriform disease may be evident. ${ }^{9}$ Mammography therefore tends to underestimate the size of DCIS, although this discrepancy is less than $20 \mathrm{~mm}$ in $80-85 \%$ of the cases if state-of-the-art mammography, including magnification views, is used. $^{10}$ It is therefore important to sample widely, and especially to sample for microscopic examination those portions of the tissue immediately adjacent to the overt radiological microcalcifications, as non-calcifying DCIS may be present more peripherally. For the same reason, it is essential to sample the margins widely. Insufficient sampling, by merely targeting calcifications on specimen slice X-ray, will result in a tendency to underestimate the size of the DCIS and also the presence of disease close to, or at, the margins of excision.

It is certainly the case that the assessment of histological DCIS size may be challenging. Ductal carcinoma in situ is a unicentric proliferation, typically involving one duct system..$^{10}$ Many pathologists seem to misinterpret areas of DCIS within a specimen as multiple foci of disease, seemingly without taking into account the three-dimensional pattern of the breast duct system. However, thorough examination with a stereoscopic technique of 60 mastectomy specimens showed a multifocal distribution (a gap of $4 \mathrm{~cm}$ or more between foci) in only one case. ${ }^{11}$ It is the case that $8 \%$ of DCIS had more than $10 \mathrm{~mm}$ 'gaps', most commonly seen in low grade disease, whereas poorly differentiated (high grade) DCIS tended to have a continuous growth pattern. Nevertheless, even if such gaps are present in an individual case, the size of DCIS reported should be the overall size of the disease process, rather than reporting individually the size of each focus.

Extremely helpful guidelines on specimen handling and reporting, including methods for the estimation of DCIS size have been outlined in the recent College of American Pathologists protocol document. ${ }^{12}$ This reminds the pathologist that if the DCIS extends to the margins of an orientated specimen (Figure 1), a minimum size can be given from the overall size of the tissue received. In other cases, large blocks can be invaluable particularly if taken along the maximum radiological extent of the calcification in the specimen X-ray (with the provisos noted above) and the maximum dimension of DCIS can then often be measured on a single large slide. Alternately, the size of DCIS can be assessed by the summation of the disease from continuous histological slides from an individual specimen slice, thus mapping the disease process. It can be helpful in such cases to either photograph or draw the specimen/specimen slices and annotate these with the site of each block, or indeed do the same on the specimen slice X-rays. If the maximum dimension of the DCIS lies across a number of slices of the specimen, this can be estimated by the assessment of the number of consecutive slices bearing the process, ${ }^{12}$ although this is inherently less accurate than direct measurement, due to estimation of the thickness of the specimen slices. Another alternative method has been described, which includes the calculation of the number of blocks bearing DCIS and multiplying this by either 0.3 or 0.4 , the latter having been reported to give a more accurate estimate. ${ }^{13,14}$ 


\section{Pathological features}

The microscopic heterogeneity of DCIS has led to the development of a number of systems for classification. Historically, DCIS has been classified on the basis of architectural pattern of the proliferation, including comedo, cribriform, micropapillary, solid or mixed subtypes. This system of categorization provides some information regarding likely extent of disease; for example, micropapillary DCIS is recognized to more often be multiquadrant $(71 \%)$ than comedo-type disease (8\%). ${ }^{15}$ Presentation may also be different, with comedo-type DCIS presenting most commonly mammographically compared with cribriform disease that is more commonly identified symptomatically, in some series. ${ }^{15}$ However, the reproducibility of this system of categorization based on growth pattern alone is problematic; lesions most frequently (62\%) show a mixture of architectures, which is seen almost twice as often as the second most commonly seen pattern (solid, $31.9 \%) .{ }^{16}$ In addition, even an individual duct space may show an architectural pattern that is difficult to categorize. Typically, therefore, newer systems tend to be based on nuclear grade, ${ }^{12,17}$ which is less commonly mixed $(15.7 \%),{ }^{16}$ some also incorporating the presence or absence of luminal necrosis. ${ }^{18}$

High-grade DCIS is formed from large, pleomorphic cells, often with multiple prominent nucleoli (Figure 1). Of particular assistance in classification is the assessment of the size of the nuclei compared with adjacent normal cells, typically either normal epithelial or red blood cells. The nuclei of high-grade DCIS are typically more than 2.5 red blood cells in diameter ${ }^{12}$ (Figure 2). Mitoses may be frequent. This grade of DCIS is often of solid architecture and tends not to show polarization of the cells and frequently bears central (comedo type) necrosis with or without associated microcalcifica-

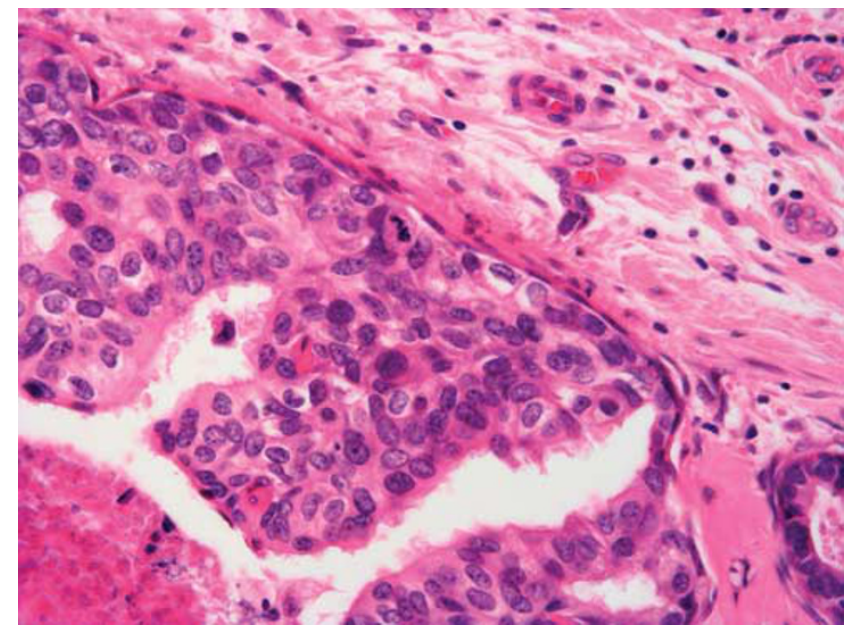

Figure 2 High-grade ductal carcinoma in situ (DCIS) seen as neoplastic cells more than 2.5 erythrocytes in diameter with clumped chromatin, some with multiple, prominent nucleoli. Central comedo-type necrosis is also present (bottom left). tion. Comedo necrosis may also, however, be seen in association with other architectural patterns (eg cribriform DCIS). The term 'comedo DCIS' is widely used in historical series but does not confer either a specific grade, or architecture, to the lesion and there is no consensus in the literature regarding the amount of central necrosis required, so reproducibility as a category of DCIS is questionable.

Conversely, low-grade DCIS is formed from evenly spaced small, regular cells with round monotonous nuclei. These are typically $1.5-2$ red blood cells in diameter $^{12}$ (Figure 3). The cells have well-defined cell boundaries and show polarization, for example, around micropapillae or cribriform structures. Mitoses are sparse and chromatin is usually finely dispersed. Nucleoli are typically not prominent.

Intermediate-grade DCIS is diagnosed when the lesion cannot be assigned to the high or low nuclear grade categories. The growth pattern is most often solid or cribriform and there is usually a degree of polarization. The nuclei show moderate pleomorphism, less than that seen in the high-grade cell disease but lack the monotony and regularity of size and spacing of the low-grade form. Perhaps not surprisingly, given the lack of precise features for categorization of DCIS as being of intermediate grade, this group has the poorest agreement in the UK National Health Service Breast Screening Programme (NHS BSP) External Quality Assurance (EQA) scheme with a $\kappa$ value of 0.23 , whereas the agreement for classification as high-grade disease is moderate $(0.51){ }^{19}$

Despite the additional effort that is required to obtain better reproducibility of grading of DCIS, as noted above one advantage of classifying DCIS according to cytonuclear grade is that there is less commonly a variation within an individual lesion. ${ }^{16}$ Indeed, genetic studies suggest that low- and-high grade DCIS have different alterations, in keeping

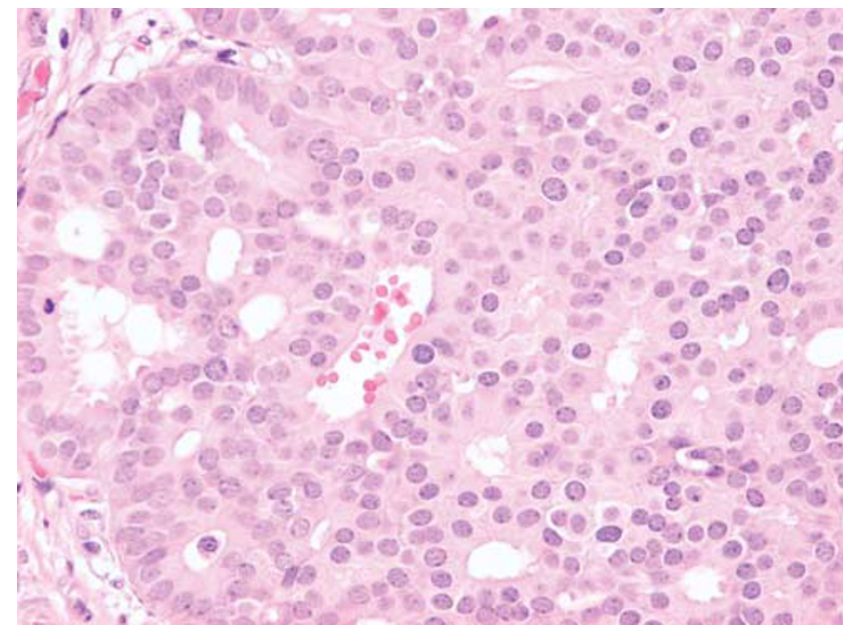

Figure 3 Low-grade ductal carcinoma in situ (DCIS) of cribriform architecture. Small nuclei (predominantly 1.5-2 erythrocytes in diameter), some with prominent nucleoli, are seen with mild pleomorphism. 
with the concept that this is a group of diseases. ${ }^{20}$ It should be noted, however, that an alternate finding is that a mixture of grades and biomarker expression may frequently be seen within an individual case of DCIS. ${ }^{21}$ How this can be reconciled with numerous data indicating that the cytonuclear grade of DCIS remains constant in progression from in situ to invasive, and indeed metastatic, disease, ${ }^{22}$ is difficult. Similarly, the genetic homology between DCIS and invasive carcinoma ${ }^{23,24}$ that is reported is also contrary to the argument that an individual case frequently shows the heterogeneity that we recognize between lesions in different patients.

Grade of DCIS in surgical excisions relates to risk of local recurrence (see below) and to be relatively reproducible; ${ }^{19,25}$ the histological grade of DCIS in core biopsy specimens is also clinically relevant with regard to the likelihood of there being radiologically unsuspected invasion. ${ }^{26}$ If, for example, a patient has more than 40 flecks of calcification on mammogram and core biopsy demonstrates highgrade DCIS, the risk of a radiologically occult invasive focus is $48 \%{ }^{26}$ Such patients may benefit from sentinel lymph nodes procedure at the time of primary breast surgery, rather than awaiting the discovery of invasive disease and requiring a second surgical procedure. Conversely, if axillary lymph nodes have been surgically removed and are found to bear metastatic tumor cells in a case in which only DCIS has been identified in the breast, clearly an invasive focus, although small, has been missed.

\section{Prognosis}

Grade of DCIS is related to the likelihood of progression to invasive carcinoma and the rapidity with which this is likely to occur. ${ }^{27}$ Although radiotherapy reduces the risk of local recurrence of disease by approximately half, ${ }^{28,29}$ a number of prognostic markers of DCIS, in addition to cytonuclear grade, have been identified in randomized clinical trials and in other series. ${ }^{28,30-34}$ The architectural growth pattern of the DCIS, the presence of necrosis, young age of patient and symptomatic detection have been reported to be poor prognostic factors for DCIS and to correlate with likelihood of local recurrence of disease, as has larger lesion size and in particular the presence of involved margins. ${ }^{28,30-34}$

Thus, the presently recognized pathological prognostic factors for local recurrence of DCIS, nuclear grade, presence/absence of necrosis and architectural pattern(s), size/extent of DCIS and distance to excision margins/margin status should all be included in the pathology report. Use of a synoptic report is one technique that is widely used to ensure that all relevant features are included and allows for standardization of reporting of DCIS lesions. One such standardized proforma has been produced by the College of American Pathologists. ${ }^{12}$

\section{Differential diagnosis}

Reassuringly, despite the variability in microscopic appearance of DCIS, reproducibility of diagnosis is substantial, as shown in the UK NHS BSP EQA scheme $(\kappa$ value $=0.76) .^{19}$ However, the differential diagnosis of DCIS includes other intraductal epithelial proliferations and lobular in situ neoplasia.

Atypical ductal hyperplasia $(\mathrm{ADH})$ is a lesion showing some, but not all, of the features of DCIS. ${ }^{17,35}$ Atypical ductal hyperplasia is diagnosed on the basis of architectural and cytological features but, in essence, if cellular changes of low-grade DCIS occupy two or more duct spaces, this should be reported as low-grade DCIS; if less extensive the process is classified as ADH. High-grade proliferations should be reported as high-grade DCIS, regardless of size or number of duct spaces involved. ADH does not have a high cytonuclear grade.

As typical of a clonal process, DCIS in general shows homogeneity of staining with a variety of markers; this can be valuable in diagnosis. Usual epithelial hyperplasia, conversely, commonly shows heterogeneity of expression, for example, of basal cytokeratins ${ }^{36}$ and generally also of estrogen receptor (ER) ${ }^{37}$ Low-grade DCIS is a luminal proliferation and shows uniform negativity for basal cytokeratins (CK5 and CK14) but high-grade DCIS, in particular, may rarely be of 'basal' phenotype. ${ }^{38,39}$ Notably, however, the expression of basal cytokeratins in high-grade DCIS may be heterogeneous and focal (Figure 4) and should not be mistaken for the mosaic pattern typically seen in usual epithelial hyperplasia.

No markers are of assistance in distinguishing low-grade DCIS from ADH, which requires an assessment of the extent of the process and the cytological features; some authorities believe that these are in fact the same process, as shown by

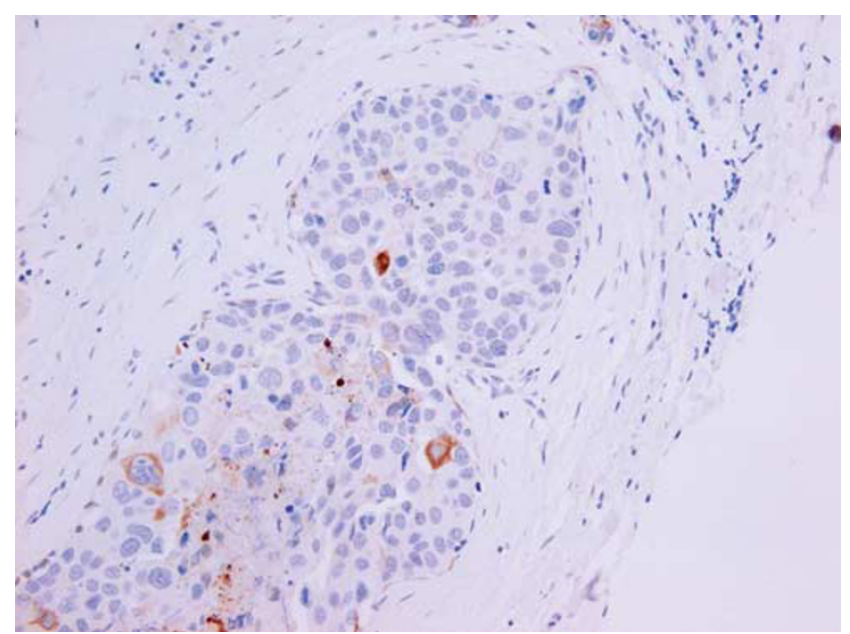

Figure 4 Cytokeratin 5/6 immunohistochemical-stained section of a case of high-grade ductal carcinoma in situ (DCIS); only occasional neoplastic cells show cytoplasmic reactivity. 
morphological, genetic and immunohistochemical similarities.

It can be difficult on rare occasions to distinguish low-grade solid DCIS from lobular carcinoma in situ (LCIS). Features in favor of DCIS are greater cellular cohesion with more evident cell borders and lack of intracytoplasmic lumina. E-cadherin immunohistochemistry can be helpful, but it should also be noted that combinations of both processes may exist and also that morphologically unequivocal LCIS may, rarely, express E-cadherin ${ }^{40}$ and ductal lesions may have reduced expression.

Microinvasive carcinoma is a lesion that has had varied definitions over time but which is now generally accepted as a lesion in which one or more separate foci of invasion are present, none of which measures more than $1 \mathrm{~mm}$ in diameter. ${ }^{12,17}$ This rare lesion typically arises within high-grade DCIS and is very unusual in low-grade disease. ${ }^{41}$ However, highgrade DCIS may extend into the lobules as so-called 'cancerization' that may closely mimic microinvasive, or invasive (ie $>1 \mathrm{~mm}$ ), carcinoma and be misdiagnosed as such. Examination of the block at deeper levels may be helpful in recognizing the lobular architecture of cancerization of lobules or of sclerosing adenosis. Search for adenosis elsewhere in the specimen may also highlight the presence of this process in the specimen and avoid a misdiagnosis. Immunohistochemistry can assist in the identification of a myoepithelial layer around islands of such atypical epithelial cells; thus smooth muscle myosin, smooth muscle actin and p63, and even basement membrane markers, such as laminin and type IV collagen, may be valuable.

\section{Conclusion}

It is the responsibility of the pathologist not only to correctly diagnose DCIS, but also to provide important prognostic information, as for invasive carcinoma. Factors that should be included in a histological report of a case of DCIS include cytonuclear grade, presence or absence of necrosis, size of the lesion and distance to margins. As described, these typically require even more diligent and thorough macroscopic specimen handling than cases of invasive breast carcinoma, in additional to careful microscopic assessment. There is abundant research being undertaken on the profiles of precursor lesions of invasive breast cancer, but as yet no biomarkers have been convincingly shown, and validated, to predict the behavior of DCIS. ${ }^{42,43}$ It remains the duty of the histopathologist to provide clinically important pathological information that guides patient treatment.

\section{Disclosure/conflict of interest}

The authors declare no conflict of interest.

\section{References}

1 Meijnen P, Gilhuijs KG, Rutgers EJ. The effect of margins on the clinical management of ductal carcinoma in situ of the breast. J Surg Oncol 2008;98:579-584.

2 Waldman FM, DeVries S, Chew KL, et al. Chromosomal alterations in ductal carcinomas in situ and their in situ recurrences. J Natl Cancer Inst 2000;92:313-320.

3 Sakorafas GH, Farley DR. Optimal management of ductal carcinoma in situ of the breast. Surg Oncol 2003;12:221-240.

4 MacDonald HR, Silverstein MJ, Mabry H, et al. Local control in ductal carcinoma in situ treated by excision alone: incremental benefit of larger margins. Am J Surg 2005;190:521-525.

5 Sigal-Zafrani B, Lewis JS, Clough KB, , et al., Institut Curie Breast Study Group. Histological margin assessment for breast ductal carcinoma in situ: precision and implications. Mod Pathol 2004;17:81-88.

6 Dillon MF, Mc Dermott EW, O’Doherty A, et al. Factors affecting successful breast conservation for ductal carcinoma in situ. Ann Surg Oncol 2007;14:1618-1628.

7 Neuschatz AC, DiPetrillo T, Steinhoff M, et al. The value of breast lumpectomy margin assessment as a predictor of residual tumor burden in ductal carcinoma in situ of the breast. Cancer 2002;94:1917-1924.

8 Cheng L, Al-Kaisi NK, Gordon NH, et al. Relationship between the size and margin status of ductal carcinoma in situ of the breast and residual disease. J Natl Cancer Inst 1997;89:1356-1360.

9 Holland R, Hendriks JH. Microcalcifications associated with ductal carcinoma in situ: mammographicpathologic correlation. Semin Diagn Pathol 1994;11: 181-192.

10 Holland R, Hendriks JH, Vebeek AL, et al. Extent, distribution, and mammographic/histological correlations of breast ductal carcinoma in situ. Lancet 1990;335:519-522.

11 Faverly DR, Burgers L, Bult P, et al. Three dimensional imaging of mammary ductal carcinoma in situ: clinical implications. Semin Diagn Pathol 1994;11:193-198.

12 Lester SC, Bose S, Chen Y-Y, , et al., for the Members of the Cancer Committee, College of American Pathologists. Protocol for the examination of specimens from patients with ductal carcinoma in situ of the breast. Arch Pathol Lab Med 2009;133:15-25.

13 Dadmanesh F, Fan X, Dastane A, et al. Comparative analysis of size estimation by mapping and counting number of blocks with ductal carcinoma in situ in breast excision specimens. Arch Pathol Lab Med 2009;133:26-30.

14 Grin A, Horne G, Ennis M, et al. Measuring extent of ductal carcinoma in situ in breast excision specimens: a comparison of 4 methods. Arch Pathol Lab Med 2009;133:31-37.

15 Bellamy CO, McDonald C, Salter DM, et al. Noninvasive ductal carcinoma of the breast: the relevance of histologic categorization. Hum Pathol 1993;24: 16-23.

16 Quinn CM, Ostrowski JL. Cytological and architectural heterogeneity in ductal carcinoma in situ of the breast. J Clin Pathol 1997;50:596-599.

17 Pathology Reporting of Breast Disease, NHS BSP Publication No 58. Sheffield. January 2005. (http:// www.cancerscreening.nhs.uk/breastscreen/publications/nhsbsp58.html). 
18 Silverstein MJ, Poller DN, Waisman JR, et al. Prognostic classification of breast ductal carcinoma-in-situ. Lancet 1995;345:1154-1157.

19 Ellis IO, Coleman D, Wells C, et al. Impact of a National External Quality Assurance Scheme for Breast Pathology in the United Kingdom. J Clin Pathol 2006;59: 138-145.

20 Roylance R, Gorman P, Hanby A, et al. Allelic imbalance analysis of chromosome $16 \mathrm{q}$ shows that grade I and grade III invasive ductal breast cancers follow different genetic pathways. J Pathol 2002;196: 32-36.

21 Allred DC, Wu Y, Mao S, et al. Ductal carcinoma in situ and the emergence of diversity during breast cancer evolution. Clin Cancer Res 2008;14:370-378.

22 Millis RR, Barnes DM, Lampejo OT, et al. Tumour grade does not change between primary and recurrent mammary carcinoma. Eur J Cancer 1998;34:548-553.

23 Buerger $\mathrm{H}$, Otterbach F, Simon R, et al. Different genetic pathways in the evolution of invasive breast cancer are associated with distinct morphological subtypes. J Pathol 1999;189:521-526.

24 Boecker W, Buerger H, Schmitz K, et al. Ductal epithelial proliferations of the breast: a biological continuum? Comparative genomic hybridization and high-molecular-weight cytokeratin expression patterns. J Pathol 2001;195:415-421.

25 Badve S, A'Hern RP, Ward AM, et al. Prediction of local recurrence of ductal carcinoma in situ of the breast using five histological classifications: A comparative study with long follow-up. Human Pathol 1998;29: 915-923.

26 Bagnall MJ, Evans AJ, Wilson AR, et al. Predicting invasion in mammographically detected microcalcification. Clin Radiol 2001;56:828-832.

27 Page DL, Dupont WD, Rogers LW, et al. Continued local recurrence of carcinoma 15-25 years after a diagnosis of low grade ductal carcinoma in situ of the breast treated only by biopsy. Cancer 1995;76: 1197-1200.

28 EORTC Breast Cancer Cooperative Group; EORTC Radiotherapy Group, Bijker N, Meijnen P, Peterse JL, et al. Breast-conserving treatment with or without radiotherapy in ductal carcinoma-in-situ: ten-year results of European Organisation for Research and Treatment of Cancer randomized phase III trial 10853 - a study by the EORTC Breast Cancer Cooperative Group and EORTC Radiotherapy Group. J Clin Oncol 2006;24:3381-3387.

29 UK Coordinating Committee on Cancer Research Ductal Carcinoma in situ Working Party. Radiotherapy and tamoxifen in women with completely excised ductal carcinoma in situ of the breast in the UK, Australia, and New Zealand: randomised controlled trial. Lancet 2003;362:95-102.
30 Di Saverio S, Catena F, Santini D, et al. 259 Patients with DCIS of the breast applying USC/Van Nuys prognostic index: a retrospective review with long term follow up. Breast Cancer Res Treat 2008;109:405-416.

31 Vargas C, Kestin L, Go N, et al. Factors associated with local recurrence and cause-specific survival in patients with ductal carcinoma in situ of the breast treated with breast-conserving therapy or mastectomy. Int J Radiat Oncol Biol Phys 2005;63:1514-1521.

32 Ringberg A, Nordgren $\mathrm{H}$, Thorstensson $\mathrm{S}$, et al. Histopathological risk factors for ipsilateral breast events after breast conserving treatment for ductal carcinoma in situ of the breast-results from the Swedish randomised trial. Eur J Cancer 2007;43: 291-298.

33 Fisher ER, Dignam J, Tan-Chiu E, et al. Pathologic findings from the National Surgical Adjuvant Breast Project (NSABP) eight-year update of Protocol B-17: intraductal carcinoma. Cancer 1999;86: 429-438.

34 Sahoo S, Recant WM, Jaskowiak N, et al. Defining negative margins in DCIS patients treated with breast conservation therapy: the University of Chicago experience. Breast J 2005;11:242-247.

35 Page DL, Dupont WD, Rogers LW, et al. Atypical hyperplastic lesions of the female breast. A long-term follow-up study. Cancer 1985;55:2698-2708.

36 Otterbach $\mathrm{F}$, Bànkfalvi $\mathrm{A}$, Bergner $\mathrm{S}$, et al. Cytokeratin 5/6 immunohistochemistry assists the differential diagnosis of atypical proliferations of the breast. Histopathology 2000;37:232-240.

37 Shoker BS, Jarvis C, Sibson DR, et al. Oestrogen receptor expression in the normal and pre-cancerous breast. J Pathol 1999;188:237-244.

38 Livasy CA, Perou CM, Karaca G, et al. Identification of a basal-like subtype of breast ductal carcinoma in situ. Hum Pathol 2007;38:197-204.

39 Bryan BB, Schnitt SJ, Collins LC. Ductal carcinoma in situ with basal-like phenotype: a possible precursor to invasive basal-like breast cancer. Modern Pathol 2006;19:617-621.

40 Acs G, Lawton TJ, Rebbeck TR, et al. Differential expression of E-cadherin in lobular and ductal neoplasms of the breast and its biologic and diagnostic implications. Am J Clin Pathol 2001;115:85-98.

41 Bianchi S, Vezzosi V. Microinvasive carcinoma of the breast. Pathol Oncol Res 2008;14:105-111.

42 Cornfield DB, Palazzo JP, Schwartz GF, et al. The prognostic significance of multiple morphologic features and biologic markers in ductal carcinoma in situ of the breast: a study of a large cohort of patients treated with surgery alone. Cancer 2004;100:2317-2327.

43 Nofech-Mozes S, Spayne J, Rakovitch E, et al. Prognostic and predictive molecular markers in DCIS: a review. Adv Anat Pathol 2005;12:256-264. 